\title{
Considerações sobre o uso de evidências científicas em tempos de pandemia: o caso da COVID-19
}

\author{
Considerations on the use of scientific evidences in times of a \\ pandemic: the case of COVID-19
}

Daniel Marques Mota ${ }^{\mathrm{l}, *}$ (iD

Ricardo de Souza

Kuchenbecker" iD
' Agência Nacional de Vigilância Sanitária (Anvisa), Brasília, DF, Brasil

" Programa de Pós-graduação em Epidemiologia, Universidade Federal do Rio Grande do Sul, Porto Alegre, RS, Brasil

\footnotetext{
* E-mail: dmarques2003@yahoo.com.br
}

Recebido: 13 abr 2020

Aprovado: 22 abr 2020

\section{RESUMO}

Fazer o melhor uso das evidências disponíveis sobre medicamentos e terapias não farmacológicas no contexto da pandemia da COVID-19 é fundamental para minimizar os sofrimentos e salvar vidas. Este debate objetivou apresentar considerações sobre o conceito de evidência, hierarquia das evidências e os tipos de evidências científicas, buscando aplicação no contexto da pandemia da COVID-19, no que tange ao uso de terapias para prevenção e tratamento da doença. Inicialmente, fizemos uma breve introdução sobre o tema, destacando a existência de dúvidas quanto ao uso de vários medicamentos, bem como se aqueles disponíveis para combater outras doenças podem ser seguros e eficazes no tratamento da COVID-19. Em seguida, apresentamos algumas definições sobre evidência, reforçando que uma definição exata depende do contexto em que será usada, podendo, inclusive, ter uma conotação abrangente ou restritiva. Na sequência, mencionamos que as evidências são classificadas em uma ordem hierárquica, ilustrada por meio de uma pirâmide, conforme o desenho do estudo empregado, um dos marcadores importantes para definir a qualidade da evidência. É dado destaque à evidência advinda da opinião de especialista, a qual está fundamentada em crenças construídas com base em teoria e aprendizagem não sistemática. Logo a seguir, recorremos a conceitos básicos sobre três tipos de evidências científicas (evidências diretas, indiretas e preliminares) para explicar as divergências entre opiniões de especialistas. Concluímos com comentários e reflexões sobre a necessidade de definir critérios razoavelmente aceitáveis para uso de evidências, por ora disponíveis, em tempos de pandemia, a exemplo da COVID-19.

PALAVRAS-CHAVE: COVID-19; Efeitos dos Fármacos; Medicina Baseada em Evidências; Pandemias; SARS-CoV-2

\begin{abstract}
Making better use of available evidence on drugs and non-pharmacological therapies in the context of the COVID-19 pandemic is critical for minimizing suffering and saving lives. This debate aimed to present considerations about the concept of evidence, the hierarchy of evidence and the types of scientific evidence, seeking application in the context of the COVID-19 pandemic, with regard to the use of therapies for prevention and treatment of the disease. Initially, we made a brief introduction on the topic, highlighting the existence of doubts regarding the use of various drugs, as well as whether those available to combat other diseases can be safe and effective in the treatment of COVID-19. Then, we present some definitions about evidence, reinforcing that an exact definition depends on the context in which it will be used, and may even have a broad or restrictive connotation. Next, we mention that the evidence is classified in a hierarchical order, illustrated by means of a pyramid, according to the design of the study employed, one of the important markers to define the quality of the evidence. Emphasis is given to the evidence from the expert opinion, which is based on beliefs built on the basis of theory and non-systematic learning. Soon after, we resorted to basic concepts about three types of scientific evidence (direct, indirect and preliminary evidence) to explain the divergences between expert opinions. We conclude with comments and reflections on the need to define reasonably acceptable criteria for the use of evidence, for now available, in times of a pandemic, such as COVID-19.
\end{abstract}

KEYWORDS: COVID-19; Drug Effects; Evidence-Based Medicine; Pandemics; SARS-CoV-2 


\section{INTRODUÇ̃̃O}

O uso de medicamentos e de outras terapias não farmacológicas para prevenir ou tratar doenças baseando-se nas melhores evidências científicas disponíveis obtidas por meio de pesquisas sistemáticas é amplamente aceito, tanto como uma boa prática clínica como para diretrizes e políticas em saúde. No entanto, há situações graves e complexas em saúde pública de ocorrência inesperada e de rápida disseminação geográfica que, ao longo da sua evolução, não contam, efetivamente, com evidências produzidas a partir dessas pesquisas ${ }^{1,2,3}$.

Atualmente, com a pandemia da COVID-19, uma doença sem tratamento específico e causada pelo coronavírus da Síndrome Respiratória Aguda Grave 2 (SARS-CoV-2) ${ }^{2,3,4}$, tem-se levantado dúvidas quanto ao uso de vários medicamentos, bem como se aqueles disponíveis para combater outras doenças podem ser seguros e eficazes no seu tratamento. Nessas condições estão os fármacos inibidores da enzima conversora da angiotensina e os bloqueadores dos receptores da angiotensina; os anti-inflamatórios não esteroides, como ibuprofeno; os antiparasitários, como hidroxicloroquina (associado ou não à azitromicina), cloroquina, nitazoxanida e ivermectina; os antirretrovirais, como lopinavir/ritonavir; os análogos de nucleotídeos, como remdesivir, e o plasma convalescente $\mathrm{e}^{1,5,6,7}$. Terapias nutricionais, como a administração de vitaminas A, D e C e o uso de zinco e selênio, também são ainda consideradas sem efeito demonstrado na prevenção e tratamento da COVID-196.

A ausência de evidências científicas robustas sobre uso de medicamentos e de terapias não farmacológicas em pacientes com COVID-19 gera incertezas nos processos decisórios clínicos e de saúde pública e potenciais consequências graves da pandemia para a população, o sistema de saúde e a economia. Por exemplo: em termos de decisão clínica, a falta de evidências faz com que um grande número de pacientes receba medicamentos em situações de uso compassivo e de uso off label com base em suas propriedades antivirais ou anti-inflamatórias obtidas de estudos in vitro ${ }^{8}$.

O objetivo deste debate foi apresentar considerações sobre o conceito de evidência, a hierarquia das evidências e os tipos de evidências científicas, buscando aplicação no contexto da pandemia da COVID-19, no que tange ao uso de terapias para prevenção e tratamento da doença. Enfatiza as evidências produzidas por fontes referidas no contexto da pirâmide das evidências, dando especial atenção, aquelas caracterizadas como opinião de especialistas.

\section{0 que é evidência?}

Evidência pode ser definida como as informações ou fatos que são obtidos sistematicamente (ou seja, obtidos de maneira replicável, observável, crível e verificável) para uso na tomada de decisões ou em julgamentos ${ }^{9}$. A Rede de Evidência de Saúde (Health Evidence Network) da Organização Mundial da Saúde (World Health Organization) define evidência como resultados de pesquisas e de outros conhecimentos que possam ser úteis para a tomada de decisões em saúde pública e na assistência médica ${ }^{10}$. É possível, no entanto, que a definição exata de evidência dependa do contexto em que será usada, podendo, inclusive, ter uma conotação mais abrangente ou restritiva ${ }^{10}$.

Em um contexto de saúde pública, as evidências podem assumir várias formas, a exemplo de um resultado de teste laboratorial para confirmação de caso da COVID-19 ou uma declaração de óbito que comprove a causa de morte do paciente. Outras formas de evidências advêm de estudos científicos ou da opinião de especialistas, os quais podem variar tanto na credibilidade em ajudar na tomada de decisões clínicas como na identificação de fatores capazes de influenciar a aplicabilidade de algo que é comprovadamente seguro e eficaz, quando da sua utilização em cenários geográficos ou institucionais específicos ${ }^{10}$.

A noção de evidência com conotação mais abrangente é conhecida como coloquial, enquanto aquela de caráter mais restritiva é denominada científica. Fora do mundo acadêmico, prevalece a definição de evidência coloquial, a qual é mais sensível ao contexto decisório. Isto significa dizer que evidência é "qualquer coisa que estabeleça um fato ou dê razão para acreditar em algo" ${ }^{10}$. É mais provável que os gestores públicos utilizem a definição coloquial de evidência nas suas decisões, muito embora o movimento de tomada de decisão baseado em evidências tenha gerado maior consideração pelas formas científicas de evidências ${ }^{10}$.

Um exemplo de definição de evidência com conotação mais restritiva é aquela proposta por Davis ${ }^{11}$ e que pode ser aplicada no contexto de incertezas sobre o uso de medicamentos no manejo clínico da COVID-19. Para o propósito do autor, "evidência" significa informações sobre relações causais entre intervenções passadas (causas) e seus resultados (efeitos) ${ }^{11}$. Essas relações causais são estabelecidas por certos tipos de estudos científicos evidenciados na pirâmide das evidências ${ }^{12}$.

\section{A hierarquia das evidências}

Os cientistas buscam utilizar métodos sistemáticos e reproduzíveis para produzir evidências de qualidade. Essas evidências são classificadas em uma ordem hierárquica, ilustrada por meio de uma pirâmide, conforme o desenho ou delineamento do estudo empregado, um dos marcadores importantes para definir a qualidade da evidência' ${ }^{12}$ (Figura).

A escolha do desenho do estudo para produzir a melhor evidência depende da pergunta de pesquisa a ser respondida ${ }^{12}$, bem como da factibilidade das estratégias utilizadas para respondê-la. Estão no topo ou perto do topo da hierarquia, os ensaios clínicos randomizados (ECR), considerados a melhor abordagem para responder perguntas sobre eficácia e segurança do tratamento de doenças. Na literatura médica, os ECR são referidos como o "padrão ouro" entre as fontes de evidências para estabelecer relações causais" ${ }^{11}$. 


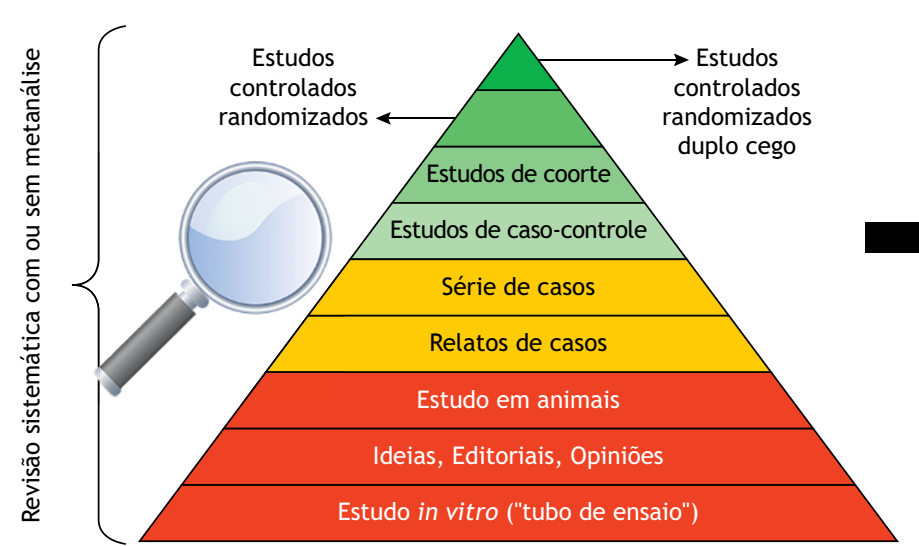

(A)

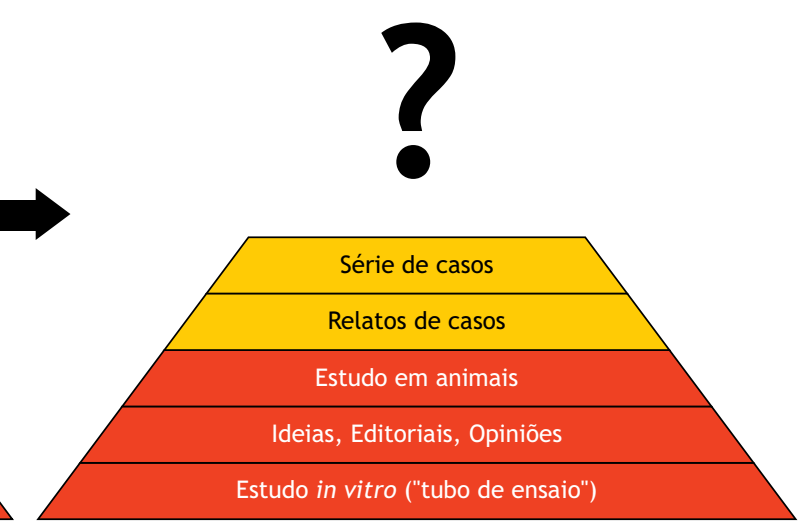

(B)

(A): representa um modelo de pirâmide das evidências tradicional aplicado a diferentes doenças com tratamentos comprovados, em termos de segurança e eficácia em situações de não emergências em saúde pública.

(B): representa um modelo de pirâmide das evidências em situações raras, a exemplo da COVID-19, principalmente, na sua fase inicial de aparecimento dos primeiros casos. É importante mencionar que com o avançar da ciência, ao longo da evolução da pandemia da COVID-19, é esperado que sejam produzidas novas evidências científicas originadas de estudos previstos no topo do modelo A.

Fonte: Adaptada de Bigby ${ }^{12}$ e Murad et al. ${ }^{13}$.

Figura. A pirâmide das evidências em dois contextos de saúde pública.

No contexto de uma emergência em saúde pública, ensaios clínicos pragmáticos baseados no efeito de tratamentos são uma alternativa útil aos ECR explanatórios que, ao provar determinado conceito, frequentemente lançam mão de critérios de inclusão e exclusão que limitam a validade externa (reprodutibilidade) dos seus achados ${ }^{14}$.

Os ensaios clínicos pragmáticos correspondem a uma das três categorias de desenhos de estudos que compõe o que se conhece como "evidência do mundo real"15. A Food and Drug Administration (FDA) define evidência do mundo real como sendo a evidência clínica sobre o uso e os possíveis benefícios ou riscos de um produto médico derivado da análise de "dados do mundo real”, os quais são dados relacionados ao estado de saúde do paciente e/ou à prestação de cuidados de saúde coletados rotineiramente de várias fontes ${ }^{16}$.

São esperados avanços no uso da "evidência do mundo real", haja vista que foi promulgada a Lei de Curas do Século XXI (21st Century Cures Act - Cures Act), de 31 de dezembro de 2016, pelo Congresso norte-americano, que determinou à FDA a criação de um marco referencial e diretrizes regulatórias para avaliar seu potencial uso para fins de aprovação de novas indicações de medicamentos já aprovados e para o cumprimento dos requisitos pós-aprovação ${ }^{17}$. Há autores, no entanto, que questionam o uso de "evidência do mundo real" para aprovações de novos medicamentos em substituição ao rigor metodológico de um ECR ${ }^{18}$.

Ressalta-se que a presença de certas limitações metodológicas de um ECR (ou de outro tipo de estudo), como imprecisão (amplo intervalo de confiança das estimativas de efeito) e inconsistência (presença de vieses, como ocultação da randomização e cegamento do estudo), podem afetar a qualidade das evidências geradas ${ }^{13}$.
A hierarquia na qualidade (força) das evidências, um princípio importante das Práticas em Saúde Baseadas em Evidências, atribui grande valor às revisões sistemáticas com ou sem metanálises de vários estudos, principalmente, as que incluem os $\mathrm{ECR}^{12}$. No modelo proposto na Figura, as revisões sistemáticas, que costumam constar no topo da pirâmide como preconizado por diferentes autores ${ }^{19}$, são usadas como uma lupa através da qual outros tipos de estudos devem ser observados, ou seja, avaliados e aplicados ${ }^{13}$.

Do lado oposto ao ECR, a opinião de um especialista, que forma a base da pirâmide, pode ser eventualmente valiosa, principalmente em condições raras, nas quais o especialista tem mais experiência num contexto de série de casos ou quando outras formas de evidência científica não estão disponíveis ${ }^{12}$.

A ordem da hierarquia das evidências tem sido amplamente discutida, alterada e, às vezes, contestada ${ }^{12}$, resultando em várias versões da pirâmide ${ }^{19}$. Essa hierarquia das evidências não deve ser interpretada como um fenômeno linear, ou seja, como uma escala que vai de "bom" a "ruim"12. A qualidade e relevância das evidências devem ser contextualizadas e consideradas, principalmente, em situações raras e graves, como a da pandemia da COVID-19. Assim, um grande estudo de coorte bem conduzido pode ser mais confiável do que um pequeno ECR que apresenta limitações metodológicas, a exemplo daquelas referidas anteriormente. Da mesma forma, um pequeno ECR de qualidade moderada que lida com o problema exato do paciente (por exemplo: psoríase palmoplantar), provavelmente, seja mais útil do que um grande ECR, que aborda um problema diferente ou mais amplo (por exemplo: psoríase) ${ }^{12}$.

A hierarquia das evidências deve considerar o contexto situacional representado seja pela magnitude dos agravos, seja pela qualidade das evidências disponíveis, o que pode ser avaliado 
mediante estratégias como a Classificação da avaliação, desenvolvimento e valoração das recomendações (Grading of Recommendations, Assessment, Development and Evaluations - Grade). Essa ferramenta analisa aspectos como: limitações metodológicas dos estudos disponíveis, risco de viés, inconsistência, evidência indireta, imprecisão, viés de publicação, magnitude do efeito, gradiente de dose-resposta e confundimento residual ${ }^{20}$.

Ao longo da curva da pandemia da COVID-19, as evidências que estão na base da pirâmide (parte B da Figura) são as que têm orientado as decisões clínicas para prevenir e tratar a doença, com destaque para a opinião de especialistas. Tais fontes de evidência são menos preferidas porque implicam em elevado grau de incerteza e necessidade de avaliação mais criteriosa sobre benefícios e riscos na prática clínica ${ }^{13}$.

\section{A opinião dos especialistas}

Desde o surgimento dos primeiros casos da COVID-19, na cidade de Wuhan (província de Hubei), na China, em dezembro de $2019^{3}$, até o momento (20 de abril de 2020), a opinião dos especialistas, como autoridades, cientistas e médicos, sobre o uso de medicamentos para prevenir e tratar a doença tem prevalecido diante da falta ou carência de evidências científicas produzidas por pesquisas sistemáticas. Essa opinião está fundamentada nas crenças dos especialistas e é formada por teoria e aprendizagem não sistemática ${ }^{11}$.

As crenças teoricamente fundamentadas são geradas mediante dedução de premissas, na maior parte das vezes baseadas em pesquisas sobre o impacto positivo de uma terapia medicamentosa frente à doença ${ }^{11}$. Por exemplo, um médico recorre a medicamentos existentes para tratar a doença. Ele acredita que o uso da hidroxicloroquina associada à azitromicina pode reduzir o número de óbitos de pacientes com COVID-19 moderada ou grave. A base para essa crença é o ensaio clínico não randomizado aberto feito com 42 pacientes hospitalizados com COVID-19 na França, apontando que a adição de azitromicina à hidroxicloroquina resultou em uma diminuição mais rápida da carga viral em comparação com o tratamento apenas com hidroxicloroquina ${ }^{21}$. Neste contexto, não são possíveis análises sobre a eficácia e a segurança dos medicamentos em relação a desfechos relevantes, como redução de complicações associadas à doença ou à mortalidade.

As crenças baseadas na aprendizagem não sistemática são frequentemente uma mistura de intuição e bom senso fundamentada na experiência pessoal, na cultura organizacional, bem como em informações adquiridas de forma não sistemática sobre as experiências ou crenças de outros profissionais e, muitas vezes, são combinadas com a teoria ${ }^{11}$. Considerando o cenário retratado anteriormente, esse mesmo médico, a partir de conversas com outros clínicos da mesma instituição que obtiveram êxitos no uso da hidroxicloroquina associada à azitromicina, passa a prescrever essa associação para seus pacientes. Isso é um exemplo de crença baseada na aprendizagem não sistemática ${ }^{11}$.
Nenhuma teoria ou aprendizagem não sistemática corresponde a uma evidência conclusiva em si e totalmente confiável para decidir sobre tratamentos seguros e eficazes ${ }^{22}$, sendo necessária sua confirmação por meio de estudos que estão no topo da pirâmide. É importante mencionar que o sistema de saúde está cheio de tratamentos utilizados com base mais em hábitos ou crenças muito fortes do que em evidência científica ${ }^{22}$. Tratamentos que muitas vezes não fazem bem algum e que, por vezes, podem acarretar dano ${ }^{22}$.

A opinião dos especialistas nas suas decisões em contexto de emergência em saúde pública pode ser orientada por Protocolos Clínicos e Diretrizes Terapêuticas (PCDT). Esses documentos obedecem a princípios e métodos de análise de evidências científicas que consideram critérios de eficácia, segurança, efetividade e custo-efetividade das intervenções em saúde ${ }^{23}$. Os PCDT contemplam, por exemplo, a definição do tema, caracterização das perguntas norteadoras, incluindo incertezas sobre melhores práticas, potencial para melhorar os resultados de saúde, bem como considerações sobre redução de iniquidades na saúde, entre outros aspectos ${ }^{23}$.

Mesmo no contexto de escassas evidências sobre a doença, a elaboração de PCDT pode reunir informações necessárias para reduzir a variabilidade de condutas clínicas, de emprego de medidas terapêuticas ineficazes, reduzindo o risco de ocorrência de reações adversas, e, por conseguinte, dos resultados em saúde obtidos $^{24}$. Outra vantagem dos PCDT é minimizar a influência de terceiros nas decisões clínicas tomadas por especialistas ${ }^{23}$.

Salienta-se, no entanto, que as informações contidas nos PCDT devem ser adaptadas a cada paciente específico com base no julgamento profissional, considerando as necessidades do paciente, os recursos disponíveis, o surgimento de novas evidências, bem como qualquer outra circunstância única ${ }^{24}$. Essas informações não devem ser usadas para substituir ou anular o julgamento de um médico qualificado ${ }^{25}$.

O uso de terapias em pacientes com a COVID-19 baseado em evidências científicas

Até então, algumas das potenciais terapias para prevenir ou tratar a COVID-19 são incertas do ponto de vista das evidências científicas disponíveis, permitindo afirmar que - até o presente momento - não há qualquer tratamento que traga mais benefícios do que riscos à saúde humana. Essa condição de incerteza tem produzido diferenças de opinião entre muitos especialistas quanto ao tratamento dos pacientes em estágios graves, moderados ou leves da doença.

Em situações não emergenciais de saúde pública, seria aconselhável aguardar o surgimento das evidências científicas que resultassem no desenvolvimento do medicamento e, por conseguinte, todo o rito regulatório necessário para sua disponibilização à população. Essa jornada, que vai desde a ideia original até o lançamento de um produto acabado, é um processo complexo que pode levar de 12 a 15 anos e custar mais de US\$ 1 bilhão ${ }^{26}$. 
No entanto, diante da pandemia da COVID-19, que tem ceifado milhares de vidas pelo mundo e desafiado a ciência, é importante entendermos os argumentos por trás das opiniões divergentes dos especialistas, recorrendo a conceitos básicos sobre três tipos de evidências científicas ${ }^{27}$ e seus potenciais usos nas decisões clínicas para prevenir e tratar doentes com a COVID-19.

Em primeiro lugar, as evidências diretas são informações científicas de qualidade suficiente para serem incorporadas à tomada de decisão em humanos, provenientes de estudos que avaliaram a terapia de interesse diretamente na doença em questão, ou seja, a COVID-1927. Até o momento, existem poucos estudos concluídos em pacientes com a COVID-19 e os que existem constituem evidências com baixo grau de certeza ${ }^{7}$. Ademais, é importante mencionar que um único estudo raramente fornecerá evidências suficientes $^{22}$ para orientar definitivamente as escolhas de tratamento de pacientes com a COVID-19. A suposta relação causal entre o medicamento e os desfechos clínicos esperados é reforçada pela frequência de tal observação oriunda de mais estudos $\operatorname{clínicos}^{28}$.

Em segundo lugar, as evidências indiretas são informações científicas de qualidade oriundas de estudos que não avaliaram diretamente a terapia de interesse na COVID-19, mas que provieram de doenças semelhantes ${ }^{27}$. Por exemplo: as evidências de estudos advindas da síndrome respiratória do oriente médio (MERS-Cov), doença ocasionada por coronavírus, são consideradas mais diretas do que as da influenza e, por sua vez, são menos indiretas do que aquelas oriundas de outras doenças respiratórias ${ }^{27}$. Este tipo de evidência, que caracteriza os medicamentos que já estão disponíveis à população para o tratamento de outras doenças, é um dos primeiros recursos a que os pesquisadores vêm recorrendo para encontrar a cura da COVID-19. Exemplos de alguns desses medicamentos foram citados na primeira seção deste debate.
Por último, as evidências preliminares são informações científicas de estudos pré-clínicos feitos com o vírus da COVID-19, mas que ainda não são tão relevantes para a tomada de decisões na clínica, como estudos experimentais em animais e em culturas de células in vitro ${ }^{27}$. Também são consideradas como evidências preliminares os estudos iniciais em pessoas com a COVID-19 que não atendem a determinadas características metodológicas ${ }^{27}$, como a alocação aleatória dos pacientes em dois grupos (intervenção e controle), garantindo que sejam o mais semelhantes possível em todos os fatores conhecidos e desconhecidos ${ }^{22}$. Os estudos iniciais em humanos permitem identificar uma correlação estatística, mas não determinam causalidade ${ }^{29}$. 0 objetivo das evidências preliminares é gerar hipóteses para que pesquisadores possam continuar avançando na identificação de terapias eficazes e seguras para prevenir e tratar pacientes com a COVID-1927.

O Quadro apresenta a classificação de estudos científicos de fármacos com possibilidades de benefícios clínicos para pacientes com a COVID-19, de acordo com os tipos de evidências discutidos anteriormente.

As divergências de opiniões dos especialistas sobre o uso de vários medicamentos e de outras terapias não farmacológicas em pacientes com a COVID-19 devem-se à existência, até o momento, de apenas evidências indiretas e preliminares com um nível muito baixo de certeza (isto é, não é possível inferir se determinado medicamento é ou não eficaz no tratamento da COVID-19), tornando necessário que a interpretação delas e o uso em decisões clínicas sejam feitos com muito cuidado ${ }^{27}$. Como regra geral, esses dois tipos de evidências científicas não permitem que sejam feitas recomendações fortes sobre terapias ${ }^{27}$ para tratamento da COVID-19, de modo que, nesses casos, a avaliação caso a caso pelo médico poderá influenciar no manejo do doente.

Quadro. Classificação de estudos científicos de fármacos com possibilidades de benefícios clínicos para pacientes com a COVID-19, segundo os tipos de evidências.

\begin{tabular}{|c|c|c|c|}
\hline Fármaco & $\begin{array}{l}\text { Classe } \\
\text { terapêutica* }\end{array}$ & Estudos concluídos ou em fase de desenvolvimento mencionados na literatura & $\begin{array}{l}\text { Tipo de } \\
\text { evidência }\end{array}$ \\
\hline \multirow{3}{*}{ Remdesivir } & \multirow{3}{*}{$\begin{array}{l}\text { Análogo de } \\
\text { nucleotídeos** }\end{array}$} & Ensaios clínicos randomizados em desenvolvimento ${ }^{\mathrm{a}, \mathrm{b}}$ & Direta $^{5}$ \\
\hline & & Relato de três casos por meio de protocolo de uso compassivo do medicamento a & \multirow{2}{*}{ Preliminar } \\
\hline & & Estudo em células in vitro contra o coronavírus da Síndrome Respiratória Aguda Grave 2 (SARS-CoV-2) & \\
\hline \multirow{2}{*}{ Cloroquina } & \multirow{2}{*}{$\begin{array}{l}\text { Antiprotozoário } \\
\text { (P01BA01) }\end{array}$} & Ensaios clínicos durante surto na China (dados não disponíveis) ${ }^{a}$ & Direta $^{\S}$ \\
\hline & & Estudo em células in vitro contra SARS-CoV-2a & Preliminar \\
\hline \multirow{3}{*}{ Hidroxicloroquina } & \multirow{3}{*}{$\begin{array}{l}\text { Antiprotozoário } \\
\text { (P01BA02) }\end{array}$} & Ensaios clínicos randomizados em desenvolvimento ${ }^{b}$ & Direta $^{5}$ \\
\hline & & Ensaio clínico aberto não randomizado (associado ou não à azitromicina) ${ }^{c}$ & \multirow{2}{*}{ Preliminar } \\
\hline & & Estudo em células in vitro contra SARS-CoV-2 ${ }^{\mathrm{a}}$ & \\
\hline \multirow{3}{*}{$\begin{array}{l}\text { Lopinavir/ } \\
\text { Ritonavir }\end{array}$} & \multirow{3}{*}{$\begin{array}{l}\text { Antivirais de } \\
\text { uso sistêmico } \\
\text { (J05AR10) }\end{array}$} & Ensaio randomizado, controlado e aberto ${ }^{\mathrm{a}, \mathrm{b}}$ & \multirow{3}{*}{ Preliminar } \\
\hline & & Estudo de coorte retrospectivo e não randomizado ${ }^{b}$ & \\
\hline & & Relato de casos e série de $\operatorname{casos}^{a}$ & \\
\hline \multirow{3}{*}{ Nitazoxanida } & \multirow{3}{*}{$\begin{array}{l}\text { Antiprotozoário } \\
\text { (P01AX11) }\end{array}$} & Ensaio clínico randomizado, duplo-cego, controlado por placebo em desenvolvimento ${ }^{d}$ & Direta $^{5}$ \\
\hline & & Estudo em células in vitro contra SARS-CoV-2 ${ }^{\mathrm{a}, \mathrm{b}}$ & Preliminar \\
\hline & & $\begin{array}{l}\text { Três ensaios clínicos randomizados e controlados realizados com pacientes acometidos de } \\
\qquad \text { Influenza (dados não disponíveis) }{ }^{\text {a }}\end{array}$ & Indireta \\
\hline Ivermectina & $\begin{array}{l}\text { Anti-helmíntico } \\
\text { (P02CF01) }\end{array}$ & Estudo em células in vitro contra SARS-CoV-2 $2^{\mathrm{e}}$ & Preliminar \\
\hline
\end{tabular}

Fonte: Elaborado pelos autores a partir dos estudos publicados por: a McCreary et al. ${ }^{1}$, bSanders et al. ${ }^{30}$, ${ }^{\mathrm{C} G a u t r e t}$ et al. ${ }^{21}$, ${ }^{\mathrm{d}} \mathrm{ReDO}{ }^{31} \mathrm{e}{ }^{\mathrm{e}} \mathrm{Caly}$ et al. ${ }^{32}$.

* Classe terapêutica, conforme a classificação Anatômico Terapêutico Químico (Anatomical Therapeutic Chemical - ATC) ${ }^{33}$.

** Não existe sua classificação na ATC.

${ }^{\S} \mathrm{O}$ tipo de evidência poderá ser alterado, a depender das limitações metodológicas dos estudos, nestes casos, sendo classificados como evidência preliminar. 
As incertezas quanto as evidências indiretas e preliminares, também, recaem sobre a dose "adequada" dos medicamentos com potenciais usos no tratamento de pacientes com a COVID-19. A título ilustrativo, à medida que a dose de um medicamento é aumentada, seus benéficos podem não gerar mais o efeito desejado, vindo a ser superados pelo aparecimento de reações adversas que comprometam a saúde do doente ${ }^{22}$.

Cabe mencionar que a ausência de evidência não significa que uma terapia não possa ser eficaz ${ }^{34}$. Significa apenas que ainda não sabemos. No entanto, é razoável não realizar estudos se não houver uma base teórica suficiente para que um benefício seja esperado. Portanto, quanto mais irracional é uma intervenção, mais fácil é descartá-la sem ter que recorrer a estudos científicos $^{34}$. Novamente, a utilização de estratégias de avaliação sistemática das evidências como o Grade podem ser úteis em contextos de elevado grau de incerteza ${ }^{20}$.

\section{CONCLUSÕES}

Na impossibilidade de prever exatamente o que se sucederá ao longo do percurso da pandemia da COVID-19, que já produziu 168.500 óbitos no mundo $(20 / 04 / 2020 \text { - 15h38m36s })^{35}$, e diante da ausência de qualquer tratamento aprovado por agências reguladoras para a doença, as decisões clínicas para minimizar os sofrimentos e salvar vidas, até o momento, têm sido tomadas com base nas opiniões dos especialistas.

Nessas condições, para que se construa um melhor cenário de decisão clínica e de saúde pública, faz-se necessário considerar, pelo menos, os seguintes aspectos: i) reunir sempre que possível a combinação do maior número de evidências para a tomada de decisão, que preferencialmente deve ter a participação dos pacientes e seus familiares; ii) escolher a opção terapêutica que tende a ser a mais adequada de acordo com as circunstâncias clínicas individuais e com os valores e as preferências dos pacientes e seus familiares ${ }^{22,36}$; e iii) dado que apenas a evidência nunca é suficiente para tomar decisões clínicas, avaliar a relação entre benefícios e riscos, a carga associada e os custos envolvidos em uma decisão, e, ao fazê-la, considerar, também, a situação (socioeconômica) dos pacientes ${ }^{36}$. Tais cuidados podem contribuir nas escolhas de terapias mais racionais, evitando esgotá-las para os tratamentos de doenças com eficácias comprovadas.
Na ausência de evidências claras e confiáveis sobre os riscos e benefícios de tratamentos, estas incertezas devem ser compartilhadas com os pacientes ${ }^{22}$, condição que pressupõe habilidades nem sempre disponíveis entre os médicos. No entanto, independentemente do que venha a ocorrer, a decisão final do médico compartilhada com o paciente ou familiares deve ser profundamente respeitada, pois é esperado que ele tenha feito o uso dos melhores recursos e evidências que estavam ao seu alcance para minimizar os sofrimentos e salvar vidas.

Como muitas vezes as decisões precisam ser tomadas em situações de emergências em saúde pública nas quais há falta ou carência de evidência científica publicada, as opiniões de especialistas são frequentemente empregadas ${ }^{37}$. Muitas delas fundamentadas em crenças construídas com base em teoria e aprendizagem não sistemática, cujas fontes de informações, no contexto da pandemia da COVID-19, têm sido as evidências indiretas e preliminares.

Nessa jornada de decisão é importante considerar alguns dos princípios basilares da Medicina hipocrática, como: i) favorecer e não prejudicar (primo non nocere, primeiramente não fazer o mal) que significa estar escolhendo o mal menor; ii) abster-se de tentar procedimentos inúteis; e iii) o dever de dedicar lealdade prioritária ao paciente ${ }^{38}$. Cabe ainda lembrar que a conduta médica deve: "curar quando possível, mas aliviar sempre" 38 .

Portanto, para garantir uma adequação razoavelmente aceitável, o desenvolvimento de uma lista de verificação da qualidade dos dados de evidências advindas da opinião de especialistas ${ }^{37}$ e de diretrizes apontando em que condições podem ser utilizadas para fundamentar as decisões clínicas é essencial, e mais pesquisas nessa direção precisam ser uma prioridade ${ }^{37}$. Mesmo em cenários dessa natureza, os PCDT, ainda que baseados em escassas evidências e necessidade de revisões a serem feitas quase que diariamente para incorporação de novas informações científicas, podem reduzir a variabilidade das condutas clínicas e a avaliação dos resultados dos tratamentos empregados. Talvez, avaliar o peso a ser atribuído a cada tipo de evidência, por ora disponível, permitindo que cada uma dela contribua adequadamente para a decisão final $^{37}$ necessite constar nesse documento orientador.

\section{REFERÊNCIAS}

1. McCreary EK, Pogue JM. Coronavirus disease 2019 treatment: a review of early and emerging options. Open Forum Infect Dis. 2020;7(4):1-11. https://doi.org/10.1093/ofid/ofaa105

2. World Health Organization - WHO. Coronavirus disease 2019 (COVID-19). Situation Report 63. 23 mar 2020.

3. Pan-American Health Organization - PAHO. Folha informativa: COVID-19 (doença causada pelo novo coronavírus). Washington: Pan-American Health Organization; 2020[acesso 29 mar 2020]. Disponível em: https: / / www.paho.org/bra/index.php?option=com_ content\&view $=$ article\&id $=6101$ : covid $19 \&$ Itemid $=875$

4. Lai CC, Shih TP, Ko WC, Tang HJ, Hsueh PR. Severe acute respiratory syndrome coronavirus 2 (SARS-CoV-2) and coronavirus disease-2019 (COVID-19): the epidemic and the challenges. Int J Antimicrob Agents. 2020;55(11):1-9. https://doi.org/10.1016/j.ijantimicag.2020.105924

5. Some drugs for COVID-19. Med Lett Drugs Ther. 2020;62(1595):49-50. 
6. Zhang L, Liu Y. Potential interventions for novel coronavirus in China: a systematic review. J Med Virol. 2020;92(5):479-90. https://doi.org/10.1002/jmv.25707

7. Pan-American Health Organization - PAHO. Ongoing living update of potential COVID-19 therapeutics: summary of rapid systematic reviews. Washington: Pan-American Health Organization, 2020[acesso 17 abr 2020]. Disponível em: https://www.paho.org/en/documents/ongoing-livingupdate-potential-COVID-19-therapeutics-summary-rapidsystematic-reviews

8. Kalil AC. Treating COVID-19 off-label drug use, compassionate use, and randomized clinical trials during pandemics. JAMA. 2020. https://doi.org/10.1001/jama.2020.4742

9. Bose M. CDC coffee break: what counts as evidence? Washington: Centers for Disease Control and Prevention; 2013[acesso 11 abr 2020]. Disponível em: https://www.cdc. gov/dhdsp/pubs/docs/cb_june_2013.pdf

10. Lomas J, Culyer AJ, McCutcheon C, McAuley L, Law S. Conceptualizing and combining evidence for health system guidance. Ottawa: Canadian Health Services Research Foundation; 2005.

11. Davis KE. The limits of evidence-based regulation: the case of anti-bribery law. NYU Law Econ Res Paper. 2019;(19-42):1-52. https://doi.org/10.2139/ssrn.3490807

12. Bigby $M$. The hierarchy of evidence. In: Williams HC, Bigby M, Herxheimer A, Naldi L, Rzany B, Dellavalle RP, Furue YRM, editores. Evidence-based dermatology. 3a ed. Nottingham: Wiley-Blackwell; 2014. p. 30-32.

13. Murad MH, Asi N, Alsawas M, Alahdab F. New evidence pyramid. Evid Based Med. 2016;21(4):125-7. https://doi.org/10.1136/ebmed-2016-110401

14. Mullins CD, Whicher D, Reese ES, Tunis S. Generating evidence for comparative effectiveness research using more pragmatic randomized controlled trials. Pharmacoeconomics. 2010;28(10):969-76. https: //doi.org/10.2165/11536160-000000000-00000

15. Sun X, Tan J, Tang L, Guo JJ, Li X. Real world evidence: experience and lessons from China. BMJ. 2018;360:1-4. https://doi.org/10.1136/bmj.j5262

16. US Food \& Drug Administration - FDA. Framework for FDA's real-world evidence program. Washington: US Food \& Drug Administration; 2018.

17. Eaglstein WH. Real-world evidence: what is it and does it matter for approval of drugs? J Am Acad Dermatol. 2018;79(2):390-1. https://doi.org/10.1016/j.jaad.2018.01.040

18. Nabhan C, Klink A, Prasad V. Real-world evidence: what does it really mean? JAMA Oncol. 2019;5(6):781-3. https://doi.org/10.1001/jamaoncol.2019.0450

19. Burns PB, Rohrich RJ, Chung KC. The levels of evidence and their role in evidence-based medicine. Plast Reconstr Surg. 2011;128(1):305-10. https://doi.org/10.1097/PRS.0b013e318219c171

20. Guyatt GH, Oxman AD, Vist GE, Kunz R, Falck-Ytter Y, Alonso-Coello P et al. Grade: an emerging consensus on rating quality of evidence and strength of recommendations. BMJ. 2008;336(7650):924-6. https://doi.org/10.1136/bmj.39489.470347.AD

21. Gautret P, Lagier JC, Parola P, Hoanga VT, Meddeb L, Mailhe $M$ et al. Hydroxychloroquine and azithromycin as a treatment of COVID-19: results of an open-label nonrandomized clinical trial. Int J Antimicrob Agents. 2020:1-24. https://doi.org/10.1016/j.ijantimicag.2020.105949

22. Evans I, Thornton H, Chalmers I, Glasziou P. Avaliação de tratamentos de saúde: identificando evidências científicas para oferecer sempre o melhor para a saúde. $2 \mathrm{a}$ ed. Barueri: Manole, 2016.

23. Ministério da Saúde (BR). Guia de elaboração de protocolos clínicos e diretrizes terapêuticas. 2a ed. Brasília: Ministério da Saúde, 2019.

24. Cook DA, Pencille LJ, Dupras DM, Linderbaum JA, Pankratz VS, Wilkinson JM. Practice variation and practice guidelines: attitudes of generalist and specialist physicians, nurse practitioners, and physician assistants. PLoS ONE. 2018;13(1):1-12. https://doi.org/10.1371/journal.pone. 0191943

25. Massachusetts General Hospital - MGH. COVID-19 treatment guidance: version 2.01. Boston: Massachusetts General Hospital; 2020[acesso 16 abr 2020]. Disponível em: https://www.massgeneral.org/news/coronavirus/ treatment-guidance/inpatient-care-recommendations

26. Hughes JP, Rees S, Kalindjian SB, Philpott KL. Principles of early drug discovery. Br J Pharmacol. 2011;162(6):1239-49. https://doi.org/10.1111/j.1476-5381.2010.01127.x

27. Epistemonikos Foudation - EF. ¿Qué tratamientos sirven para prevenir o tratar el coronavirus (COVID-19)? Santiago: Epistemonikos Foudation; 2020[acesso 24 mar 2020]. Disponível em: https://es.epistemonikos.cl/2020/03/17/ que-tratamientos-sirven-para-prevenir-o-tratar-elcoronavirus-COVID-19/

28. Mota DM, Kuchenbecker RS. Causalidade em farmacoepidemiologia e farmacovigilância: uma incursão teórica. Rev Bras Epidemiol. 2017;20(3):475-86. https: / /doi.org/10.1590/1980-5497201700030010

29. Epistemonikos Foudation - EF. Enalapril, losartán y otros fármacos que actúan en el sistema renina angiotensina aldosterona: deben preocuparse los pacientes que los consumen en el contexto de la pandemia por COVID-19? Santiago: Epistemonikos Foudation; 2020[acesso 12 abr 2020]. Disponível em: https://es.epistemonikos. $\mathrm{cl} / 2020 / 03 / 28 /$ enalapril-losartan-y-otros-farmacos-queactuan-en-el-sistema-renina-angiotensina-aldosteronadeben-preocuparse-los-pacientes-que-los-consumen-enel-contexto-de-la-pandemia-por-COVID-19/

30. Sanders JM, Monogue ML, Jodlowski TZ, Cutrell JB. Pharmacologic treatments for coronavirus disease 2019 (COVID-19): a review. JAMA. 2020. https://doi.org/10.1001/jama.2020.6019

31. Repurposing Drugs in Oncology Project - ReDO. Covid19 DB: number of trials in database. Strombeek-Bever: Repurposing Drugs in Oncology Project; 2020[acesso 18 abr 2020]. Disponível em: http://www.redo-project.org/covid19db/ 
32. Caly L, Druce JD, Catton MG, Jans DA, Wagstaff KM. The FDA-approved drug ivermectin inhibits the replication of SARS-CoV-2 in vitro. Antiviral Res. 2020;178:1-4. https://doi.org/10.1016/j.antiviral.2020.1047

33. WHO Collaborating Centre for Drug Statistics Methodology - Whocc. ATC/DDD index 2020. Washington: World Health Organization; 2020[acesso 18 abr 2020]. Disponível em: https://www.whocc.no/atc_ddd_index/

34. Epistemonikos Foudation. Líquidos calientes, exposición al frío, alcohol, cocaína y otras noticias falsas sobre COVID-19. Santiago: Epistemonikos Foudation; 2020[acesso em 12 abr 2020]. Disponível em: https://es.epistemonikos. $\mathrm{cl} / 2020 / 03 / 18 /$ liquidos-calientes-exposicion-al-frio-alcoholcocaina-y-otras-noticias-falsas-sobre-COVID-19/

35. Johns Hopkins University - JHU. COVID-19 dashboard by the center for systems science and engineering (CSSE) at Johns Hopkins University. Baltimore: Johns Hopkins University; 2020[acesso 20 abr 2020]. Disponível em: https://gisanddata.maps.arcgis.com/apps/opsdashboard/ index.html\#/bda7594740fd40299423467b48e9ecf6

36. Guyatt G, Jaeschke R, Wilson MC, Montori VM, Richardson WS. What is evidence-based medicine? In: Guyatt G, Rennie D, Meade MO, Cook DJ. Users' guides to the medical literature: a manual for evidence-based clinical practice. 3a ed. Ontario: McGraw-Hill, 2015. p. 9-23.

37. Sharma T, Choudhury M, Kaur B, Naidoo B. Evidence informed decision making: the use of "colloquial evidence" at nice. Int J Technol Assess Health Care. 2015;31(3):138-46. https://doi.org/10.1017/S0266462314000749

38. Neves NC. Ética para os futuros médicos: é possível ensinar? Brasília: Conselho Federal de Medicina, 2006.

Contribuição dos Autores

Mota DM - Concepção, planejamento (desenho do estudo), análise, interpretação dos dados e redação do trabalho. Kuchenbecker RS Análise, interpretação dos dados e redação do trabalho. Todos os autores aprovaram a versão final do trabalho.

Os autores informam não haver qualquer potencial conflito de interesse com pares e instituições, políticos ou financeiros deste estudo.

Esta publicação está sob a licença Creative Commons Atribuição 3.0 não Adaptada.

Para ver uma cópia desta licença, visite http://creativecommons.org/licenses/by/3.0/deed.pt_BR. 